https://doi.org/10.22319/rmcp.v12i3.5646

Artículo

\title{
Diversidad genética y factores de virulencia de cepas de Staphylococcus aureus aisladas de la piel de ubre bovina
}

Roberto Adame-Gómez ${ }^{\text {a }}$

Jeiry Toribio-Jimenez ${ }^{\mathrm{b}}$

Natividad Castro-Alarcón ${ }^{\mathrm{c}}$

Karina Talavera-Alarcón ${ }^{\text {a }}$

Jacqueline Flores-Gavilan $^{\text {a }}$

Sandra-Alheli Pineda-Rodríguez ${ }^{\mathrm{d}}$

Arturo Ramírez-Peralta ${ }^{\text {a* }}$

${ }^{\text {a } U n i v e r s i d a d ~ A u t o ́ n o m a ~ d e ~ G u e r r e r o . ~ F a c u l t a d ~ d e ~ C i e n c i a s ~ Q u i ́ m i c o ~ B i o l o ́ g i c a s, ~ L a b o r a t o r i o ~}$ de Investigación en Patometabolismo Microbiano. Chilpancingo, Guerrero, México.

${ }^{\mathrm{b}}$ Universidad Autónoma de Guerrero. Facultad de Ciencias Químico Biológicas, Laboratorio de Microbiología Molecular y Biotecnología Ambiental. Chilpancingo, Guerrero, México.

${ }^{c}$ Universidad Autónoma de Guerrero. Facultad de Ciencias Químico Biológicas, Laboratorio de Investigación en Microbiología. Chilpancingo, Guerrero, México.

${ }^{\text {d } U n i v e r s i d a d ~ A u t o ́ n o m a ~ d e ~ G u e r r e r o . ~ F a c u l t a d ~ d e ~ C i e n c i a s ~ Q u i ́ m i c o ~ B i o l o ́ g i c a s, ~ L a b o r a t o r i o ~}$ de Investigación en Parasitología. Chilpancingo, Guerrero, México.

*Autor de correspondencia: ramirezperaltauagro@gmail.com

\section{Resumen:}

Staphylococcus aureus es un patógeno reconocido como causa de mastitis en bovinos a nivel mundial, por lo cual el objetivo de este trabajo fue determinar la presencia de Staphylococcus aureus en la piel de pezón de la ubre bovina y relacionarlo a la presencia de mastitis, así 
como determinar los factores de virulencia y la diversidad genética de las cepas. En tres establos se tomaron muestras de 250 vacas de ordeña en dos temporadas del año, estiaje y lluvias. Además, se realizó la prueba de California. Staphylococcus aureus fue aislado en agar sal y manitol e identificado bioquímicamente y confirmado con la amplificación del gen femA. Para la identificación de los factores de virulencia se usaron los genes $h l B$, mec, saK, $p v L$, tsst-1, seA, $s e B, s e C, s e D$ y seE por PCR en punto final. Para la tipificación de S. aureus, se realizó la amplificación y restricción del gen coag. La frecuencia de $S$. aureus fue $13.4 \%$. No se encontró una relación estadística entre la presencia de $S$. aureus en la piel de la ubre bovina y el desarrollo de mastitis subclínica. El gen de la enterotoxina más frecuente en las cepas fue el de la enterotoxina A. Aunque el porcentaje de tipificación es bajo, se lograron identificar dos restrictotipos que agrupan cepas aisladas de diferentes vacas, lo cual evidencia la capacidad infectocontagiosa del microorganismo.

Palabras clave: Ubre bovina, Staphylococcus aureus, Diversidad genética, Mastitis.

Recibido: 20/03/2020

Aceptado: 08/01/2021

\section{Introducción}

La mastitis bovina (MB) es la inflamación de la glándula mamaria causada en la mayoría de los casos por un microorganismo, el cual invade la ubre a través del canal del pezón ${ }^{(1,2)}$. Esta enfermedad es clasificada de acuerdo a las manifestaciones clínicas que presentan, en mastitis clínica (MC) y mastitis bovina subclínica (MSC), siendo esta última, la entidad clínica más común ${ }^{(3)}$. Aún cuando son numerosos los géneros bacterianos que causan mastitis, solamente una cantidad reducida de especies son prevalentes y constituyen un problema real de salud pública $^{(2)}$.

Staphylococcus aureus es reconocido como un patógeno a nivel mundial por causar mastitis ${ }^{(4)}$, siendo responsable aproximadamente de un tercio de los casos de mastitis clínica y subclínica en bovinos ${ }^{(5,6)}$, considerándose un problema en la industria pecuaria debido a su patogenicidad, persistencia en el ambiente, facilidad de contagio entre vaca y vaca durante el proceso de ordeño ${ }^{(2,7)}$, debido a las bajas tasas de resolución de la enfermedad con los tratamientos actuales ${ }^{(8)}$, conllevando infecciones crónicas, que persisten entre periodos de lactación, con intermitentes episodios clínicos que ocurren desde la elevación de la temperatura, grados de anorexia, la aparición de coágulos en la leche ${ }^{(1)}$, lo cual incrementa los riesgos de sacrificio del animal, el trabajo, el tratamiento y los costos de reemplazo, visitas de veterinarios, incorporación de protocolos para evitar riesgo con inversión en 
infraestructura así como la implementación de métodos de diagnóstico con mayor frecuencia ${ }^{(9)}$. Sin embargo, la mastitis no solo tiene un impacto en la economía del productor, sino también se convierte en un problema de salud pública al ser una fuente potencial de transmisión zoonótica, debido a que se ha descrito que $S$. aureus es capaz de causar enfermedad en humanos ${ }^{(10,11)}$. La mastitis bovina tiene relevancia en el contexto de las intoxicaciones alimentarias en humanos. La ingestión de productos contaminados con enterotoxinas estafilococcicas produce una intoxicación caracterizada por vómito violento y diarrea $^{(12)}$. Dentro de los factores relacionados a una infección por $S$. aureus en la glándula mamaria se encuentra la higiene tanto en el ordeño como en la ubre del animal ${ }^{(13,14)}$.

En años recientes, se ha planteado que la colonización de la piel de la ubre podría ser un factor predominante al desarrollo de mastitis por arrastre del microorganismo durante el ordeño hacia el pezón del animal; sin embargo, diversos estudios difieren en relación a esta aseveración. Se ha descrito que los casos de mastitis por $S$. aureus son causados por cepas altamente adaptadas a la glándula mamaria y que son diferentes de los aislados de piel ${ }^{(2,15)}$. Mientras otros estudios sugieren que la mayoría de $S$. aureus aislados de la piel y del canal del pezón así como de los sitios extra mamarios como vagina, narinas y piel de las fauces son genéticamente iguales a las encontradas en glándulas mamarias o leche ${ }^{(16-18)}$. Por lo anterior, el objetivo de este estudio fue determinar la frecuencia de $S$. aureus en la piel de los pezones de ubres de vacas de tres establos lecheros ubicados el sur del estado de Guerrero, México, la diversidad genética y de factores de virulencia de las cepas de $S$. aureus, así como la posible relación con mastitis bovina.

\section{Material y métodos}

\section{Establos y muestreo}

Tres establos fueron incluidos en el estudio. Basados en el permiso de los propietarios y el tamaño de la granja. Se tomó una muestra de la piel del pezón de los cuatro cuartos de las 250 vacas de ordeña, en dos temporadas del año distintas: lluvias y estiaje, teniendo un total de 500 muestras. Las muestras se tomaron con un hisopo de algodón, el cual se deslizó en cada borde del pezón y alrededor del mismo cubriendo un área de $2 \mathrm{~cm}$. Después, se realizó la prueba de California, considerando la interpretación de la prueba anteriormente descrita. Todos los establos venden la leche cruda directamente a los consumidores y una de ellas es caracterizada por ser un sitio de producción de quesos elaborados con leche cruda. En esta granja, la leche cruda es colectada durante la mañana y directamente procesada en una pequeña planta artesanal de producción de quesos. 


\section{Aislados}

Las muestras tomadas con hiposos de la piel del pezón se cultivaron en agar sal y manitol. Los aislados fueron presuntivamente identificados como $S$. aureus de acuerdo al siguiente esquema: manitol positivo, catalasa positivo, coco Gram positivos y coagulasa positivo en 6 h. Los aislamientos se conservaron a $-20{ }^{\circ} \mathrm{C}$ en caldo BHI (por sus siglas en inglés "Brain Heart Infusion") con 15\% (V/V) de glicerol.

\section{Identificación molecular de S. aureus}

Las cepas fueron cultivadas en caldo infusión cerebro corazón e incubadas a $37{ }^{\circ} \mathrm{C}$. Las cepas testigo usadas en este estudio fueron S. aureus ATCC29231 (sea), S. aureus ATCC14458 (seb), S. aureus ATCC19095 (sec), S. aureus ATCC13563 (sed), S. aureus ATCC27664 (see) y S. aureus ATCC25923 (femA, coag, hlb, sak).

El ADN total se obtuvo de $1 \mathrm{ml}$ de un cultivo de $18 \mathrm{~h}$ de todas las cepas bacterianas incluidas las cepas ATCC. Las células se sedimentaron a partir de la centrifugación de los cultivos a 10,000 rpm por 10 min y suspendidas en $300 \mu \mathrm{l}$ de buffer de lisis $(10 \mathrm{mM}$ Tris $\mathrm{HCl}, 1 \mathrm{mM}$ EDTA, pH 8,0, lisozima $1 \mathrm{mg} / \mathrm{ml}$ ) e incubadas a $37{ }^{\circ} \mathrm{C}$ por media hora o hasta la observación de viscosidad. El ADN de todas las preparaciones fue subsecuentemente extraído con fenol cloroformo y precipitado con etanol. Las muestras de ADN se diluyeron en buffer TE (10 $\mathrm{mM}$ Tris $\mathrm{HCl}, 1$ mM EDTA, $\mathrm{pH} 8.0)^{(19)}$.

Una PCR en punto final del gen femA fue realizada a las cepas para la confirmación molecular de $S$. aureus con los oligonucleótidos descritos en el Cuadro 1. La mezcla final de reacción de PCR contenía $0.2 \mathrm{mM}$ de cada dNTP, $3 \mathrm{mM}$ de $\mathrm{MgCl}_{2}, 0.2 \mathrm{mM}$ de los oligonucleótidos, $1 \mathrm{U}$ taq ADN polimerasa (Amplicon, Denmark) $5 \mu \mathrm{l}$ de buffer 10X y $100 \mathrm{ng}$ de ADN como molde.

Cuadro 1: Oligonucleótidos usados para la identificación molecular, detección de genes de enterotoxinas y tipificación molecular

\begin{tabular}{clcc}
\hline $\begin{array}{c}\text { Gene (factor de } \\
\text { virulencia) }\end{array}$ & \multicolumn{1}{c}{ Secuencia (5'-3') } & TPA & Ref. \\
\hline \multirow{2}{*}{ emA } & $\begin{array}{c}\text { femaF- AAAAAAGCACATAACAAGCG } \\
\text { femaR- GATAAAGAAGAAAACCAGCAG }\end{array}$ & 130 & $(20)$ \\
\hline \multirow{2}{*}{$\operatorname{soa}$ (coagulasa) } & coaF- CGAGACCAAGATTCAACAAG & $600-$ & $(22)$ \\
& coaR- AAAGAAAACCACTCACATCA & 900 & \\
\hline \multirow{2}{*}{$s e A$ (enterotoxina A) } & seaF- TGCAGGGAACAGCTTTAGGC & 250 & $(20)$ \\
& seaR- GTGTACCACCCGCACATTGA & & \\
\hline
\end{tabular}




\begin{tabular}{|c|c|c|c|}
\hline $\operatorname{se} B($ enterotoxina $\mathrm{B})$ & $\begin{array}{l}\text { sebF- } \\
\text { ATTCTATTAAGGACACTAAGTTAGGG } \\
\text { sebR- ATCCCGTTTCATAAGGCGAGT }\end{array}$ & 400 & \\
\hline $\operatorname{seC}($ enterotoxina $\mathrm{C})$ & $\begin{array}{l}\text { secF- GTAAAGTTACAGGTGGCAAAACTTG } \\
\text { secR- } \\
\text { CATATCATACCAAAAAGTATTGCCGT }\end{array}$ & 297 & \\
\hline $\operatorname{seD}($ enterotoxina $\mathrm{D})$ & $\begin{array}{l}\text { sedF- } \\
\text { GAATTAAGTAGTACCGCGCTAAATAATA } \\
\text { TG } \\
\text { sedR- GCTGTATTTTTCCTCCGAGAGT }\end{array}$ & 492 & \\
\hline $\operatorname{seE}($ enterotoxina $\mathrm{E})$ & $\begin{array}{l}\text { seeF- } \\
\text { CAAAGAAATGCTTTAAGCAATCTTAGGC } \\
\text { seeR- CACCTTACCGCCCAAAGCTG }\end{array}$ & 480 & \\
\hline$h l B($ hemolisina $\beta)$ & $\begin{array}{l}\text { hlbF- GTGCACTTACTGACAATAGTGC } \\
\text { hlbR- GTTGATGAGTAGCTACCTTCAGT }\end{array}$ & 300 & \\
\hline$s a K$ (estafiloquinasa) & $\begin{array}{l}\text { sakF- ATCCCGTTTCATAAGGCGAGT } \\
\text { sakR- CACCTTACCGCCCAAAGCTG }\end{array}$ & 260 & $\begin{array}{l}\text { En este } \\
\text { estudio }\end{array}$ \\
\hline $\begin{array}{l}\text { mecA (Resistencia a } \\
\text { meticilina) }\end{array}$ & $\begin{array}{l}\text { mecaF- TCCAGATTACAACTTCACCAGG } \\
\text { mecaR- CCACTTCATATCTTGTAACG }\end{array}$ & 180 & $(21)$ \\
\hline $\begin{array}{l}\text { tsst-1 (toxina del } \\
\text { syndrome del shock } \\
\text { tóxico) }\end{array}$ & $\begin{array}{l}\text { tsstF- CATCTACAAACGATAATATAAAGG } \\
\text { tsstR- } \\
\text { CATTGTTATTTTCCAATAACCACCCG }\end{array}$ & 476 & $\begin{array}{l}\text { En este } \\
\text { estudio }\end{array}$ \\
\hline
\end{tabular}

TPA= tamaño del producto amplificado (pb); Ref.= referencia.

\section{Identificación de genes codificantes para factores de virulencia}

La detección de los genes $h l B$, mec, saK, pvL, tsst-1, seA, seB, seC, seD y seE codificantes para la $\beta$-hemolisina, región de resistencia a meticilina, estafiloquinasa, la toxina Panton Valentine, la toxina del síndrome del choque tóxico y enterotoxinas respectivamente, fue a partir de PCR en punto final con los oligonucleótidos descritos en el Cuadro 1. La mezcla final de reacción de PCR contenía: $0.2 \mathrm{mM}$ de cada dNTP, $3 \mathrm{mM} \mathrm{MgCl} 2,0.2 \mu \mathrm{M}$ de los oligonucleótidos, $1 \mathrm{U}$ de Taq ADN polimerasa (Ampliqon ${ }^{\circledR}$, DEN), 1X Buffer y $100 \mathrm{ng}$ de DNA como templado. Las mezclas de reacción se sometieron bajo las siguientes condiciones de amplificación: desnaturalización inicial por $5 \mathrm{~min}$, a $94{ }^{\circ} \mathrm{C}$; 30 ciclos de 30 seg a $94{ }^{\circ} \mathrm{C}$, 30 seg a $52{ }^{\circ} \mathrm{C}, 30 \mathrm{seg}$ a $72{ }^{\circ} \mathrm{C}$; y una extensión final por 5 min a $72{ }^{\circ} \mathrm{C}$ para $m e c, h l B, p v L$, tsst-1, seA y seE. Desnaturalización inicial por 5 min a $94{ }^{\circ} \mathrm{C} ; 30$ ciclos de 30 seg a $94{ }^{\circ} \mathrm{C}$, $45 \mathrm{seg} \mathrm{a} 52^{\circ} \mathrm{C}, 45 \mathrm{seg}$ a $72{ }^{\circ} \mathrm{C}$; y una extensión final por 5 min a $72{ }^{\circ} \mathrm{C}$ para $s a K$, seB, seC y $s e D^{(20,21)}$. 
La electroforesis de los de los productos de PCR se realizó en geles de agarosa al 2\% a $80 \mathrm{~V}$ durante 60 min. Los geles fueron teñidos con Midori green (Nippon Genetics®, GER) y visualizados con luz LED (Nippon Genetics®, GER) a $470 \mathrm{~nm}$.

\section{Prueba fenotípica para evidenciar la expresión del gen $h l B$}

Para demostrar la expresión de la $\beta$ hemolisina, las cepas se cultivaron por estría cruzada en agar gelosa sangre de carnero al $5 \%$, incubándose a $37{ }^{\circ} \mathrm{C}$ en tensión de $\mathrm{CO}_{2}$ por $24 \mathrm{~h}$. Las cepas que presentaron un halo de transparencia en el perímetro de las colonias se consideraron $\beta$-hemolíticas $\left(h l B^{+}\right)$. Las cepas que presentaron $\alpha$ y $\gamma$-hemolisis se consideraron $h l B^{-}$.

\section{Tipificación molecular de S. aureus}

A las cepas confirmadas molecularmente como $S$. aureus, se les realizó la amplificación del gen coag mediante PCR en punto final con los oligonucleótidos descritos en el Cuadro 1 y con la siguiente mezcla final para cada reacción de PCR: $0.2 \mathrm{mM}$ de cada dNTP, $3 \mathrm{mM}$ $\mathrm{MgCl}_{2}, 0.2 \mu \mathrm{M}$ de oligonucleótidos, $1 \mathrm{U}$ de Taq DNA polimerasa (Ampliqon®, DEN), $1 \mathrm{X}$ Buffer y $100 \mathrm{ng}$ de DNA como molde. El protocolo de PCR inicia con desnaturalización inicial de $5 \mathrm{~min}$, a $94{ }^{\circ} \mathrm{C}$; 30 ciclos de $30 \mathrm{seg}$ a $94{ }^{\circ} \mathrm{C}$, $30 \mathrm{seg}$ a $52{ }^{\circ} \mathrm{C}, 60 \mathrm{seg}$ a $72{ }^{\circ} \mathrm{C}$; y una extensión final por 5 min a $72{ }^{\circ} \mathrm{C}^{(22)}$. Los productos de PCR fueron digeridos por $2 \mathrm{~h}$ a $37{ }^{\circ} \mathrm{C}$ con $10 \mathrm{U}$ de la enzima de restricción AluI (Thermo Scientific ${ }^{\circledR}$, EE.UU.) de acuerdo al protocolo recomendado por el fabricante. Los fragmentos de restricción se detectaron por electroforesis en geles de agarosa al $2 \%$ a $70 \mathrm{~V}$ por $60 \mathrm{~min}$. Los geles se tiñeron con de Midori green (Nippon Genetics®, GER) y visualizados con luz LED (Nippon Genetics®, GER).

\section{Análisis estadístico}

Se utilizó el paquete estadístico STATA V. 12 (STATA®, EE. UU.) para calcular frecuencias simples y se utilizó la prueba estadística Ji cuadrada para posibles relaciones entre la presencia de $S$. aureus en la piel de ubre bovina y el desarrollo de mastitis subclínica, se consideran valores de $P=<0.05$ como estadísticamente significativos.

\section{Resultados y discusión}

En este estudio, del total de vacas analizadas en los dos periodos, se determinó una frecuencia de mastitis subclínica del $6.6 \%$ (33/500) y de mastitis clínica del $0.8 \%$ (4/500). La frecuencia de mastitis subclínica por establo lechero fue mayor en el establo A (12\%) en relación al B $(4 \%)$ y el C (1\%) $(P=0.001)$ (Cuadro 2). Estas diferencias en cuanto a frecuencias pueden 
estar relacionadas a la implementación o cumplimiento de estrategias bien caracterizadas para el control de infecciones intramamarias o conocido como el plan de los 5 puntos; en el cual se incluye la secuencia de ordeño, uso de guantes, cambio de toalla de papel o tela entre los cuartos de la ubre, pre sello y post sello del pezón ${ }^{(23)}$. Además, de otros factores como la presencia de infección y el tiempo de evolución de éstas ${ }^{(24)}$, la naturaleza del agente infeccioso, la paridad y el estado de lactación del animal, así como la nutrición y el ambiente en donde este se encuentre ${ }^{(25)}$. En este último punto, se conoce que la temporada de lluvias (verano) es un factor de riesgo ambiental para el desarrollo de mastitis, siendo más frecuente la aparición de casos durante esta temporada en relación a otras como el invierno ${ }^{(26)}$, mismo que fue observado en nuestro estudio, en el cual, durante el periodo de lluvias la frecuencia de mastitis subclínica fue mayor $(10.8 \%)$ con relación a la temporada de seca $(2.4 \%)(P=$ 0.001). Diversos autores coinciden en esta afirmación, considerando que la humedad podría tener un papel importante; sin embargo, también concluyen que este periodo podría coincidir con el inicio de la lactación o el término de la gestación, que por lo general debe considerarse para las vacas de primera paridad durante la temporada con el clima más deseable, en este caso el verano, durante el cual la disposición de pasto y por ende de alimento es mayor ${ }^{(27,28)}$.

Cuadro 2: Frecuencia de mastitis subclínica y clínica en relación a establos lecheros, temporal y presencia de $S$. aureus en el pezón de ubres de vacas

\begin{tabular}{|c|c|c|c|c|c|c|}
\hline \multirow[b]{2}{*}{ Característica } & \multirow[b]{2}{*}{ Total } & \multicolumn{4}{|c|}{ Prueba de california } & \multirow[b]{2}{*}{$p$} \\
\hline & & Negativo & Trazas & $\begin{array}{l}\text { Mastitis } \\
\text { subclínica }\end{array}$ & $\begin{array}{l}\text { Mastitis } \\
\text { clínica }\end{array}$ & \\
\hline \multicolumn{7}{|l|}{ Establo lechero } \\
\hline A & 200 & $8(4)$ & $164(82)$ & $24(12)$ & $4(2)$ & \multirow{3}{*}{0.001} \\
\hline B & 200 & $4(2)$ & $188(94)$ & $8(4)$ & 0 & \\
\hline $\mathrm{C}$ & 100 & 0 & 99 (99) & $1(1)$ & 0 & \\
\hline \multicolumn{7}{|l|}{ Temporal } \\
\hline Lluvias & 250 & $11(4.4)$ & $208(83.2)$ & $27(10.8)$ & $4(1.6)$ & \multirow[b]{2}{*}{0.001} \\
\hline Secas & 250 & $1(0.4)$ & $242(97.8)$ & $6(2.4)$ & 0 & \\
\hline \multicolumn{7}{|c|}{ S. aureus en ubre } \\
\hline Positivo & 67 & 0 & $66(98.5)$ & $1(1.5)$ & 0 & \multirow{2}{*}{0.106} \\
\hline Negativo & 433 & $12(2.7)$ & $385(88.9)$ & $32(7.5)$ & $4(0.9)$ & \\
\hline
\end{tabular}

Los casos de mastitis clínica solo se presentaron en el establo lechero A (2\%) y en temporada de lluvias $(1.6 \%)(P=0.001)$. En el caso del aislamiento del microorganismo de interés en la piel del pezón de la ubre de las vacas analizadas, la frecuencia de $S$. aureus a partir de la identificación del gen femA fue del $13.4 \%$ (67/500) (Figura 1, Cuadro 2). Otros marcadores son utilizados para determinar la especie de $S$. aureus como el gen de la termonucleasa $(n u c)^{(29)}$ y la región del gen del $16 \mathrm{rRNA}^{(30)}$; sin embargo, el gen $n u c$ puede ser encontrado en otras especies de Staphylococcus coagulasa positivos (S. hyicus, S. delphini, S. 
intermedius, S. pseudointermedius, S. schleiferi) y negativos ( $S$. capitis, S. caprae, $S$. epidermidis, S. warneri, S. simulans, S. carnosus, S. kloosii, S. saprophyticus) ${ }^{(31)}$, por lo cual se decidió trabajar con dicho gen, el cual está relacionado en la síntesis del peptidoglucano y tiene un alto poder de identificación para $S$. aureus resistentes a meticilina ${ }^{(32)}$.

Figura 1: Electroforesis de la amplificación del gen femA de cepas de $S$. aureus de la piel de la ubre bovina

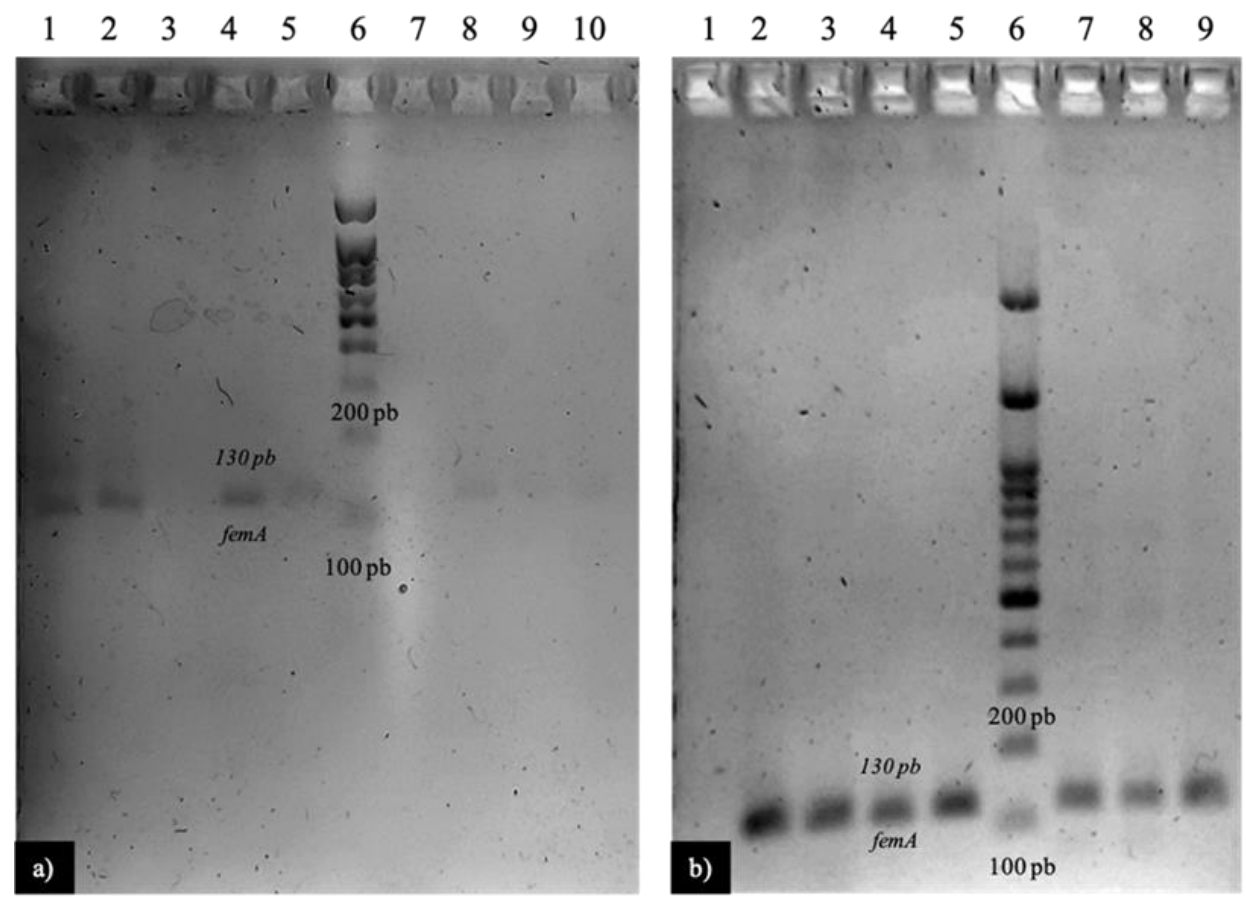

A) 1, S522 (AL); 2, S528 (AL), 3; S519 (AL) 4, S529 (AL); 5, S521 (BL); 6, MPM; 7, control negativo; 8, control positivo (S. aureus ATCC25923); 9, S517 (AL); 10, S643 (BS).

B) 1, control negativo; 2, S522 (AL); 3, S528 (AL); 4, S529 (AL); 5, S521 (BL); 6, MPM (100 PB); 7, S517 (AL); 8, S643 (BS); 9, S650 (BS);

$\mathbf{a}=$ establo $\mathrm{A} ; \mathbf{b}=$ establo $\mathrm{B} ; \mathrm{L}=$ lluvias; $\mathrm{S}=$ secas. MPM= Marcador de peso molecular de $100 \mathrm{pb}$.

En $\mathbf{B}$, las cepas débilmente positivas y positivas se volvieron a repetir y se modificaron las condiciones de electroforesis.

S. aureus fue aislado en la mayoría de cuartos lecheros que solo presentaron trazas o baja cantidad de células somáticas, y aislándose solamente en una vaca con mastitis subclínica. Determinando que no existe una relación entre los aislados de S. aureus de la piel del pezón de la ubre de las vacas con el desarrollo de cuadros de mastitis $(P=0.106)$. Diversos factores podrían explicar la ausencia de esta relación, desde el estado inmunitario del animal, el inóculo del microorganismo y las características genéticas de la cepa. Incluso en años recientes, se ha descrito el papel de la microbiota de la ubre de la vaca como un factor importante en el desarrollo de la mastitis, con la presencia de otros microorganismos que podrían actuar como antagonistas de patógenos restringiendo su multiplicación o establecimiento de la infección; por ejemplo, se ha descrito que $S$. chromogenes produce 
bacteriocinas capaces de inhibir el crecimiento de la mayoría de patógenos intramamarios ${ }^{(33,34)}$. Considerando que factores como la paridad y el estado de inmunosupresión generado durante la gestación, la lactancia, el perfil metabólico y la carga genética del animal podrían impactar positiva o negativamente en la composición de la microbiota de la ubre y por ende en la susceptibilidad de la mastitis ${ }^{(35)}$.

Aun cuando no se encontró una relación con los casos de mastitis subclínica en el estudio, se buscaron otras relaciones como la distribución de S. aureus en los tres establos lecheros (A, B y C), la cual fue del $14,11.5$ y $16 \%$ respectivamente, no observándose diferencias estadísticamente significativas $(P=0.531)$ (Cuadro 3$)$. Estos resultados podrían explicarse por la ubicuidad del microorganismo, la capacidad infectocontagiosa, así como de persistencia del mismo en el ambiente lechero ${ }^{(2)}$. Las diferencias observadas en la estacionalidad en este estudio con mayor frecuencia para $S$. aureus fue en temporada de seca $(23.6 \%, 29 / 250)$ en relación a lluvias $(3.2 \%, 8 / 250)$, podrían ser explicadas por el crecimiento acelerado de microorganismos asociados a la temperatura y la humedad de la estación ${ }^{(36)}$, así como un estado metabólico alterado, incluido algún tipo de inmunocompromiso debido a la restricción de alimento ${ }^{(35)}$, lo cual favorezca la colonización del microrganismo.

Cuadro 3: Presencia de S. aureus en el pezón de ubres de vacas en relación a establos lecheros y temporal

\begin{tabular}{lllll}
\hline Características & Total & \multicolumn{2}{c}{ S. aureus } & \multirow{2}{*}{$\boldsymbol{P}$} \\
\cline { 3 - 4 } & & Positivos & Negativos & \\
\hline Establo lechero & & $28(14.0)$ & $172(86.0)$ & \\
A & 200 & $23(11.5)$ & $177(88.5)$ & 0.531 \\
B & 200 & $16(16.0)$ & $84(84.0)$ & \\
C & 100 & & & \\
Temporal & & $8(3.20)$ & $242(96.8)$ & \multirow{2}{*}{0.001} \\
$\quad$ Lluvias & 250 & $59(23.6)$ & $191(76.4)$ & \\
$\quad$ Secas & 250 & & & \\
\hline
\end{tabular}

De igual modo no se encontró una relación estadística entre la presencia de $S$. aureus en la piel de la ubre bovina y el desarrollo de mastitis subclínica; sin embargo, se determinaron factores de virulencia que son claves tanto en el desarrollo de infecciones como de intoxicaciones alimentarias ${ }^{(37,38)}$. El gen de la enterotoxina más frecuente en las cepas de $S$. aureus fue el de la enterotoxina A (10.44 \%), destacando que no se encontraron cepas con los genes para las enterotoxinas B y C. En cuanto a toxinas, se encontraron 23 cepas con el gen de la hemolisina $\beta$ ( $34.32 \%$ ); es importante mencionar que este dato, está ajustado con la prueba fenotípica de hemolisis en sangre de carnero, lo cual se consideró debido a que los oligonucleótidos diseñados para este gen, no lo amplifican por completo y este gen es 
susceptible para la inserción de fagos y la inclusión de genes como el de la enterotoxina A y la estafiloquinasa $(\mathrm{saK})^{(39,40)}$. En este estudio, no se encontraron cepas con uno de los genes asociados a la toxina Panton Valentine.

Finalmente, solo se encontraron dos cepas con el gen $\operatorname{saK}(2.98 \%)$ y tres cepas se definieron como SARM a partir de la amplificación del gen mecA (4.47 \%) (Cuadro 4). En este sentido, diversos estudios han buscado establecer un perfil de virulencia característico de las cepas de S. aureus capaces de causar mastitis subclínica, en el supuesto, de que el microorganismo se debe adaptar a un determinado entorno al invadir la ubre bovina, los resultados han sido diversos, sin embargo, como puntos en común, convergen en que la hemolisina b y la toxina Panton Valentine, son factores de virulencia que podrían participar en el desarrollo de la mastitis subclínica, al tener como función principal, la formación de poros o lisis de leucocitos ${ }^{(2,41,42)}$. Lo que podría confirmarse con los resultados en este estudio, las cepas al tener en baja frecuencia o nula, los genes de estas toxinas (tanto el de la hemolisina como la toxina Panton Valentine) no se asociaron a casos de mastitis subclínica. No se estableció una relación de estas cepas con cuadros clínicos particulares en la ubre bovina; sin embargo, las cepas tienen importancia epidemiológica por la presencia de genes de enterotoxinas, éstas podrían contaminar por arrastre durante la ordeña de leche y en última instancia encontrarse en el producto lácteo final y ocasionar un problema de intoxicación alimentaria ${ }^{(43)}$.

Cuadro 4: Factores de virulencia de cepas de $S$. aureus aisladas de ubres de vaca

\begin{tabular}{|c|c|}
\hline Factor de virulencia & $n(\%), N=67$ \\
\hline \multicolumn{2}{|l|}{ Enterotoxinas } \\
\hline$s e A$ & $7(10.44)$ \\
\hline$s e B$ & - \\
\hline seC & - \\
\hline$s e D$ & $2(2.98)$ \\
\hline$s e E$ & $1(1.49)$ \\
\hline tsst-1 & $4(5.97)$ \\
\hline \multicolumn{2}{|l|}{ Toxinas } \\
\hline$h l B$ & $23(34.32)$ \\
\hline$p v L$ & - \\
\hline \multicolumn{2}{|l|}{ Enzimas } \\
\hline$s a K$ & $2(2.98)$ \\
\hline mecA & $3(4.47)$ \\
\hline
\end{tabular}

En cuanto a la tipificación molecular de las cepas de $S$. aureus, el porcentaje de tipificación ha presentado variaciones importantes en relación al tiempo; en los años cercanos al inicio del uso de la técnica de PCR- RFLP`s del gen coag, se logró la tipificación del $100 \%$ de las cepas $^{(44-48)}$; no obstante, en los últimos años este porcentaje disminuye hasta $40 \%{ }^{(29,30,49)}$, lo 
cual aún es más alto del porcentaje determinado en este estudio (19.47 \%) (Figura 1a). En este sentido, se deben de considerar dos eventos, en primera instancia se ha propuesto que la disminución en el porcentaje de tipificación podría estar relacionado a mutaciones en el gen de la coagulasa, que impacten en la especificidad de los oligonucleótidos planteados, pero que no afectan la actividad de la enzima, remarcando que los oligonucleótidos son los mismos que los usados en la técnica original y fueron establecidos desde $1992^{(22)}$; por otro lado, es importante comparar los resultados en relación al tipo de hospedero del cual se aisló $S$. aureus, debido a que el cambio de hospedero podría involucrar cambios importantes en la cepa incluida la variabilidad del gen coag; sin embargo, si se compara el porcentaje de tipificación en estudios de $S$. aureus en vacas, los resultados varían considerablemente entre porcentajes de tipificación ${ }^{(29,30,44,49)}$. Aunque el porcentaje de tipificación es bajo, se logró identificar un restrictotipo (400 pb, $350 \mathrm{pb}$ ) que agrupa cepas aisladas de diferentes vacas tanto del establo B (Figura 2c, carril 4) como el establo A (Figura 2b, carril 2,3 y 4; Figura 2c, carril 1,2 y 3), así como de temporada de lluvias (Figura 2b, carril 2; Figura 2c, carril 1,2 y 3 ) y de estiaje (Figura 2b, carril 3 y 4), lo cual evidencia la capacidad infectocontagiosa de S. aureus, y esto reafirma el papel de este microorganismo en casos de mastitis de tipo infeccioso, lo cual también remarca la importancia de adoptar medidas que eviten la transmisión del microorganismo en el ambiente lechero, al poder infectar al ganado bovino, alterar la calidad de la leche y el ganado debe ser considerado como una fuente de contaminación importante en la producción de derivados lácteos.

Figura 2: Electroforesis de la restricción del gen coag de cepas aisladas de la piel de la ubre bovina

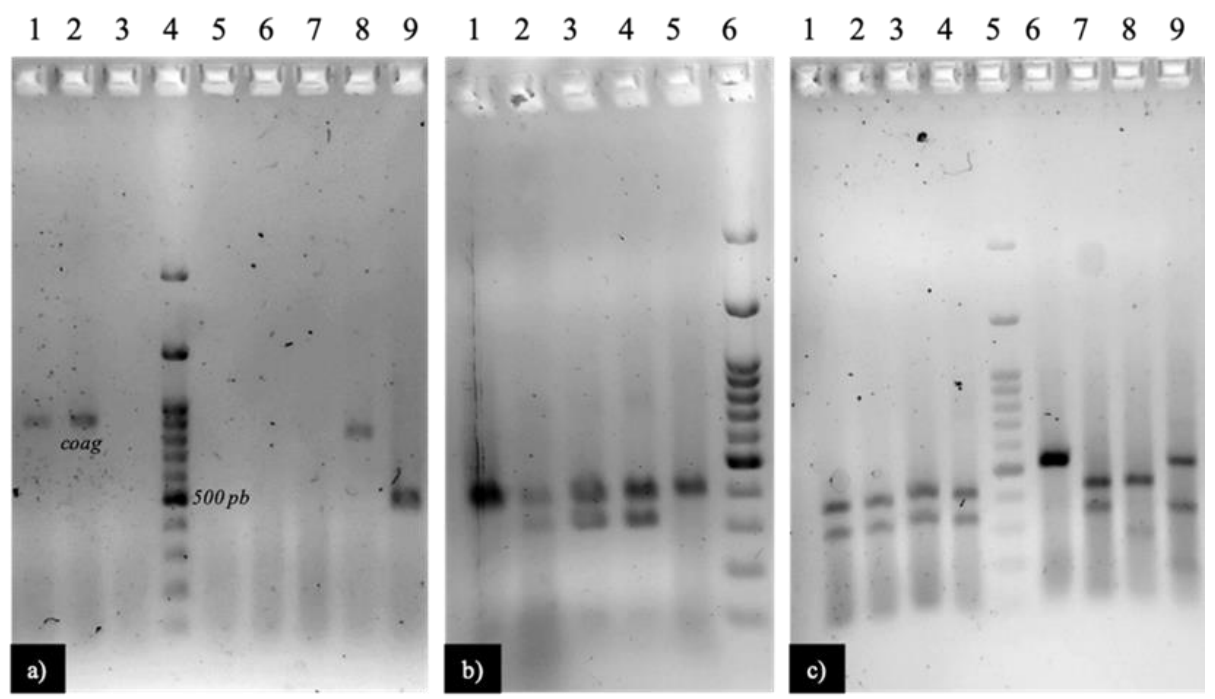
A) 1, S528; 2, testigo positivo (S. aureus ATCC25923); 3, testigo negativo; 4, MPM; 5, S519 (PL); 6, S520
(PL); 7, S530 (PL); 8, S618; 9, S673 (PS).
B) 1, S673 (PS); 2, S665 (PL); 3, 668 (PS); 4, 669 (PS); 671 (PS); 6, MPM
c) 1, S522 (PL); 2, S528 (PL); 3, S529 (PL); 4, S521 (CL); 5, MPM (100 PB); 6, S517 (PL); 7, S643 (CS); 8, S650 (CS); 9, S618 (BL).
$\mathbf{a}=$ establo A; $\mathbf{b}=$ establo $\mathrm{B}, \mathrm{L}=$ lluvias; $\mathrm{S}=$ secas. 


\section{Conclusiones e implicaciones}

En este estudio, no se encontró una relación entre la presencia de $S$. aureus en la piel del pezón de la ubre de ganado bovino con el desarrollo de mastitis; sin embargo, se determinaron cepas con genes para enterotoxinas, las cuales son un problema de salud pública. Además, se evidenció la transmisión de cepas en los establos lecheros, lo que remarca la importancia de las buenas prácticas de ordeño.

\section{Agradecimientos y conflictos de intereses}

Se les extiende un agradecimiento a todos los propietarios del ganado bovino incluido en este estudio, quienes accedieron a participar y colaboraron en el manejo del ganado durante la toma de muestra, y que además siempre externaron su preocupación por la salud de su ganado. Un agradecimiento especial a la Dra. Elvia Rodríguez Bataz, por los comentarios finales de la revisión del manuscrito.

\section{Literatura citada:}

1. Pumipuntu N, Tunyong W, Chantratita N, Diraphat P, Pumirat P, Sookrung N, et al. Staphylococcus spp. associated with subclinical bovine mastitis in central and northeast provinces of Thailand. Peer J 2019;7:e6587. doi:10.7717/peerj.6587.

2. Rainard P, Foucras G, Fitzgerald JR, Watts JL, Koop G, Middleton JR. Knowledge gaps and research priorities in Staphylococcus aureus mastitis control. Transbound Emerg Dis 2018;65:149-165.

3. Islam MA, Islam MZ, Islam M, Rahman M, Islam MT. Prevalence of subclinical mastitis in dairy cows in selected areas of Bangladesh. Trop Anim Health Prod 2012;9(1):7378.

4. Zecconi AA, Calvinho LFL, Fox K. Staphylococcus aureus intramammary infections. International Dairy Federation; 2006. Report No 408.

5. Botrel MA, Haenni M, Morignat E, Sulpice P, Madec JY, Calavas D. Distribution and antimicrobial resistance of clinical and subclinical mastitis pathogens in dairy cows in Rhône-Alpes, France. Foodborne Pathog Dis 2010;7(5):479-487.

6. Bradley AJ, Leach KA, Breen JE, Green LE, Green MJ. Survey of the incidence and aetiology of mastitis on dairy farms in England and Wales. Vet Rec 2007;160(8):253258. 
7. da Costa LB, Rajala-Schultz PJ, Hoet A, Seo KS, Fogt K, Moon BS. Genetic relatedness and virulence factors of bovine Staphylococcus aureus isolated from teat skin and milk. J Dairy Sci 2014;97(11):6907-6916.

8. Peton V, Le Loir Y. Staphylococcus aureus in veterinary medicine. Infect Genet Evol 2014;21:602-615.

9. Halasa T, Huijps K, Østerås O, Hogeveen H. Economic effects of bovine mastitis and mastitis management: A review. Vet Q 2007;29(1):18-31.

10. Balaban N, Rasooly A. Staphylococcal enterotoxins. Int J Food Microbiol 2000;61(1):110.

11. Honeyman AL, editor. Staphylococcus aureus infection and disease. New York, NY: Kluwer Acad./Plenum Publ; 2001.

12. Fetsch A, Johler S. Staphylococcus aureus as a foodborne pathogen. Curr Clin Microbiol Rep 2018;5(2):88-96.

13. Neave FK, Dodd FH, Kingwill RG, Westgarth DR. Control of mastitis in the dairy herd by hygiene and management. J Dairy Sci 1969;52(5):696-707.

14. Schreiner DA, Ruegg PL. Relationship between udder and leg hygiene scores and subclinical mastitis. J Dairy Sci 2003;86(11):3460-3475.

15. Zadoks RN, van Leeuwen WB, Kreft D, Fox LK, Barkema HW, Schukken YH, et al. Comparison of Staphylococcus aureus isolates from bovine and human skin, milking equipment, and bovine milk by phage typing, pulsed-field gel electrophoresis, and binary typing. J Clin Microbiol 2002;40(11):3894-33902.

16. Capurro A, Aspán A, Ericsson UH, Persson WK, Artursson K. Identification of potential sources of Staphylococcus aureus in herds with mastitis problems. J Dairy Sci 2010;93(1):180-191.

17. Haveri M, Hovinen M, Roslof A, Pyorala S. Molecular types and genetic profiles of Staphylococcus aureus strains isolated from bovine intramammary infections and extramammary sites. J Clin Microbiol 2008;46(11):3728-3735.

18. Mørk T, Kvitle B, Jørgensen HJ. Reservoirs of Staphylococcus aureus in meat sheep and dairy cattle. Vet Microbiol 2012;155(1):81-87.

19. Asadollahi P, Delpisheh A, Hossein MM, Azizi JF, Alikhani MY, Asadollahi K, et al. Enterotoxin and exfoliative toxin genes among methicillin-resistant Staphylococcus aureus isolates recovered from Ilam, Iran. Avicenna $\mathrm{J}$ Clin Microb Infec 2014;1(2):20208. doi:10.17795/ajcmi-20208. 
20. Jarraud S, Mougel C, Thioulouse J, Lina G, Meugnier H, Forey F, et al. Relationships between Staphylococcus aureus genetic background, virulence factors, agr groups (alleles), and human disease. Infect Immun 2002;70(2):631-341.

21. Milheiriço C, Oliveira DC, de Lencastre H. Update to the multiplex PCR strategy for assignment of mec element types in Staphylococcus aureus. Antimicrob Agents Chemother 2007;51(9):3374-3377.

22. Goh SH, Byrne SK, Zhang JL, Chow AW. Molecular typing of Staphylococcus aureus on the basis of coagulase gene polymorphisms. J Clin Microbiol 1992;30(7):1642-1645.

23. Keane OM. Symposium review: Intramammary infections - major pathogens and strainassociated complexity. J Dairy Sci 2019;102(5):4713-4726.

24. Bradley AJ. Bovine Mastitis: An evolving disease. Vet J 2002;164(2):116-128.

25. Moyes KM. Triennial lactation symposium: Nutrient partitioning during intramammary inflammation: A key to severity of mastitis and risk of subsequent diseases? J Anim Sci 2015;93(12):5586-5593.

26. Joshi S, Gokhale S. Status of mastitis as an emerging disease in improved and periurban dairy farms in India. Ann N Y Acad Sci 2006;1081(1):74-83.

27. Morse D, DeLorenzo MA, Wilcox CJ, Collier RJ, Natzke RP, Bray DR. Climatic effects on occurrence of clinical mastitis. J Dairy Sci 1988;71(3):848-853.

28. Smith KL, Todhunter DA, Schoenberger PS. Environmental mastitis: cause, prevalence, prevention. J Dairy Sci 1985;68(6):1531-1553.

29. Javid F, Taku A, Bhat MA, Badroo GA, Mudasir M, Sofi TA. Molecular typing of Staphylococcus aureus based on coagulase gene. Vet World 2018;11(4):423-430.

30. Sharma V, Sharma S, Dahiya DK, Khan A, Mathur M, Sharma A. Coagulase gene polymorphism, enterotoxigenecity, biofilm production, and antibiotic resistance in Staphylococcus aureus isolated from bovine raw milk in North West India. Ann Clin Microbiol Antimicrob 2017;16(1):65. doi:10.1186/s12941-017-0242-9.

31. Hirotaki S, Sasaki T, Kuwahara-Arai K, Hiramatsu K. Rapid and accurate identification of human-associated Staphylococci by use of multiplex PCR. J Clin Microbiol 2011;49(10):3627-3631.

32. Vannuffel P, Heusterspreute M, Bouyer M, Vandercam B, Philippe M, Gala JL. Molecular characterization of from and-based discrimination of staphylococcal species. Res Microbiol 1999;150(2):129-141. 
33. Braem G, Stijlemans B, Van Haken W, De Vliegher S, De Vuyst L, Leroy F. Antibacterial activities of coagulase-negative Staphylococci from bovine teat apex skin and their inhibitory effect on mastitis-related pathogens. J Appl Microbiol 2014;116(5):10841093.

34. Carson DA, Barkema HW, Naushad S, De Buck J. Bacteriocins of Non-aureus Staphylococci isolated from bovine milk. Appl Environ Microbiol 2017;83(17) doi:10.1128/AEM.01015-17.

35. Derakhshani H, Fehr KB, Sepehri S, Francoz D, De Buck J, Barkema HW, et al. Invited review: Microbiota of the bovine udder: Contributing factors and potential implications for udder health and mastitis susceptibility. J Dairy Sci 2018;101(12):10605-10625.

36. Hogan J, Smith KL. Managing environmental mastitis. Vet Clin North Am Food Anim Pract 2012;28(2):217-224.

37. Kadariya J, Smith TC, Thapaliya D. Staphylococcus aureus and Staphylococcal Foodborne disease: An ongoing challenge in public health. BioMed Res Int 2014;2014:1-9.

38. Tong SYC, Davis JS, Eichenberger E, Holland TL, Fowler VG. Staphylococcus aureus infections: epidemiology, pathophysiology, clinical manifestations, and management. Clin Microbiol Rev 2015;28(3):603-661.

39. van Wamel WJB, Rooijakkers SHM, Ruyken M, van Kessel KPM, van Strijp JAG. The innate immune modulators Staphylococcal complement inhibitor and chemotaxis inhibitory protein of Staphylococcus aureus are located on hemolysin-converting bacteriophages. J Bacteriol 2006;188(4):1310-1315.

40. Argudín MÁ, Mendoza MC, Rodicio MR. Food Poisoning and Staphylococcus aureus Enterotoxins. Toxins 2010;2(7):1751-1773.

41. Foster TJ. Immune evasion by staphylococci. Nat Rev Microbiol 2005;3(12):948-958.

42. van Kessel KPM, Bestebroer J, van Strijp JAG. Neutrophil-mediated phagocytosis of Staphylococcus aureus. Front immunol 2014;26(5):467. doi: 10.3389/fimmu.2014.00467.

43. Piccinini R, Cesaris L, Daprà V, Borromeo V, Picozzi C, Secchi C, et al. The role of teat skin contamination in the epidemiology of Staphylococcus aureus intramammary infections. J Dairy Res 2009;76(1):36-41.

44. Aarestrup FM, Dangler CA, Sordillo LM. Prevalence of coagulase gene polymorphism in Staphylococcus aureus isolates causing bovine mastitis. Can J Vet Res 1995;59(2):124-128. 
45. Hookey JV, Richardson JF, Cookson BD. Molecular typing of Staphylococcus aureus based on PCR restriction fragment length polymorphism and DNA sequence analysis of the coagulase gene. J Clin Microbiol 1998;36(4):1083-1099.

46. Schlegelová J, Dendis M, Benedík J, Babák V, Rysánek D. Staphylococcus aureus isolates from dairy cows and humans on a farm differ in coagulase genotype. Vet Microbiol 2003;92(4):327-334.

47. Schwarzkopf A, Karch H. Genetic variation in Staphylococcus aureus coagulase genes: potential and limits for use as epidemiological marker. J Clin Microbiol 1994;32(10):2407-2412.

48. Su C, Herbelin C, Frieze N, Skardova O, Sordillo LM. Coagulase gene polymorphism of Staphylococcus aureus isolates from dairy cattle in different geographical areas. Epidemiol Infect 1999;122(2):329-336.

49. Momtaz H, Tajbakhsh E, Rahimi E, Momeni M. Coagulase gene polymorphism of Staphylococcus aureus isolated from clinical and sub-clinical bovine mastitis in Isfahan and Chaharmahal va Bakhtiari provinces of Iran. Comp Clin Path 2011;20(5):519-522. 